\title{
Automated Local Texture and Stress Analysis in Cu Interconnects using D- STEM and Precession Microscopy
}

\author{
K.J. Ganesh*, S. Rajasekhara*, D. Bultreys**, J.P. Zhou* and P.J. Ferreira* \\ * Materials Science and Engineering Program, University of Texas at Austin, Austin, TX 78712, \\ USA \\ ** NanoMEGAS SPRL Blvd. Edmond Machtens 79, B-1080, Brussels, Belgium
}

Copper interconnect (CI) line widths continue to decrease as higher chip speeds are required. Knowledge of local texture in narrow CI lines is critical to address reliability issues like stress induced void formation and electromigration. In this regard, a novel Diffraction-Scanning Transmission Electron Microscopy (D-STEM) technique [1] has been employed to obtain local orientation information from grains as small as $3 \mathrm{~nm}$ in a completely automated manner. This technique represents a major leap in the characterization of CIs.

In this work, D-STEM has been coupled with Gatan ${ }^{\mathrm{TM}}$ STEM Diffraction Imaging (SDI) software to obtain a line scan across multiple grains along a CI line (Fig.1a). Orientation analysis was carried out to correlate local grain misorientations with thermal stresses in CIs. These stresses are caused due to differences in the coefficients of thermal expansion between CI lines and the silicon substrate. Diffraction patterns obtained from each grain (labeled one through ten in Fig. 1a) were indexed using the Automated Crystallography for TEM (ACT) software. Four of the representative diffraction patterns have been shown in Fig.1(b-e) These copper grains were found to exhibit a $<110>$ normal orientation while the $<\overline{112}>$ and the $<\overline{11}>$ type orientations were present along the length and width of the $\mathrm{CI}$ line, respectively. The elastic modulus of $\mathrm{Cu}$ is lowest along the $<100>$ direction. Therefore, sidewall copper grains would favor alignment of the $<100>$ direction along the length of the CI lines, where the stress is maximum [2]. Since the $\{111\}$ plane in copper does not contain the $<100>$ direction, the direction closest to $<100>$ available in the $\{111\}$ plane is $<112>$, which aligns along the line to minimize strain energy. This has been observed in our experiments as well. Local stresses have been calculated by applying the Objected Oriented Finite Element Method (FEM) software for microstructure analysis (OOF2) as depicted in Fig. 1(f-j).

However, the aforementioned technique becomes very tedious to automate when used to index patterns from highly off-zone axis grains. To counter such issues and obtain a more statistical analysis of texture, a complete process automation of acquisition and indexing has been carried out by integrating the D-STEM configuration with full-precession using the ASTAR ${ }^{\mathrm{TM}}$ system from NanoMEGAS. Near-kinematical diffraction patterns obtained from each pixel have been used to generate an orientation map from multiple CI lines which have been overlaid on a reconstructed bright field image (Fig. 2a).

In summary, the combination of D-STEM with SDI/ACT facilitates a way to rapidly obtain spot diffraction patterns from many nanosized grains. Furthermore, integration of D-STEM with ASTAR provides an improved method to obtain orientation and phase maps from an ensemble of nanostructures. Such information of local texture can be combined with FEM stress analysis to obtain local stress and stress gradients in narrow CI lines and address reliability issues like stress induced void formation. 
References

[1] K.J. Ganesh et al. Microsc. Microanal. 15 (Suppl. 2) (2009) 752.

[2] D. N. Lee et al. Journal of Electronic Materials 32 (2003) 1012.

[3] K.J. Ganesh et al. Scripta Materialia (2010), doi:10.1016/j.scriptamat.2010.02.016

[4] This research was supported by Semiconductor Research Corporation (SRC) under contract 2008-KJ-1854.
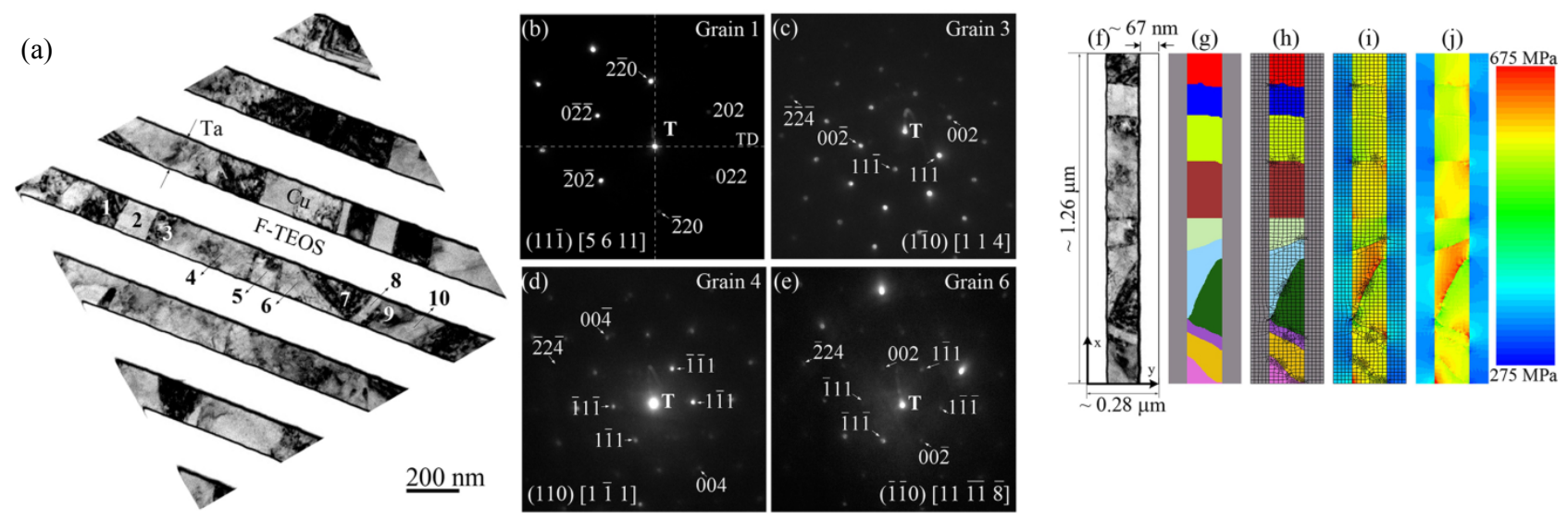

Figure 1: (a) Bright field STEM image of $120 \mathrm{~nm}$ CI lines showing a polycrystalline grain structure. Ten adjacent grains were labeled and diffraction information was obtained. (b)-(e) Diffraction patterns from grains 1, 3, 4 and 6 show that except for grain 1, the plane normal for the remaining three grains is in the $<110>$ direction, (f) Bright-field STEM image containing the CI line with ten adjacent grains on which the stress analysis was performed, (g) A color-coded replica of image (f) that contains stiffness and orientation information for each grain and the dielectric (ILD), (h) Application of a fine mesh on image ( $\mathrm{g}$ ) to divide it into finite elements, (i) Image containing the fine mesh and the FEM stress solution for every grain and the ILD, (j) Image containing the FEM stress solution without the mesh. [3]
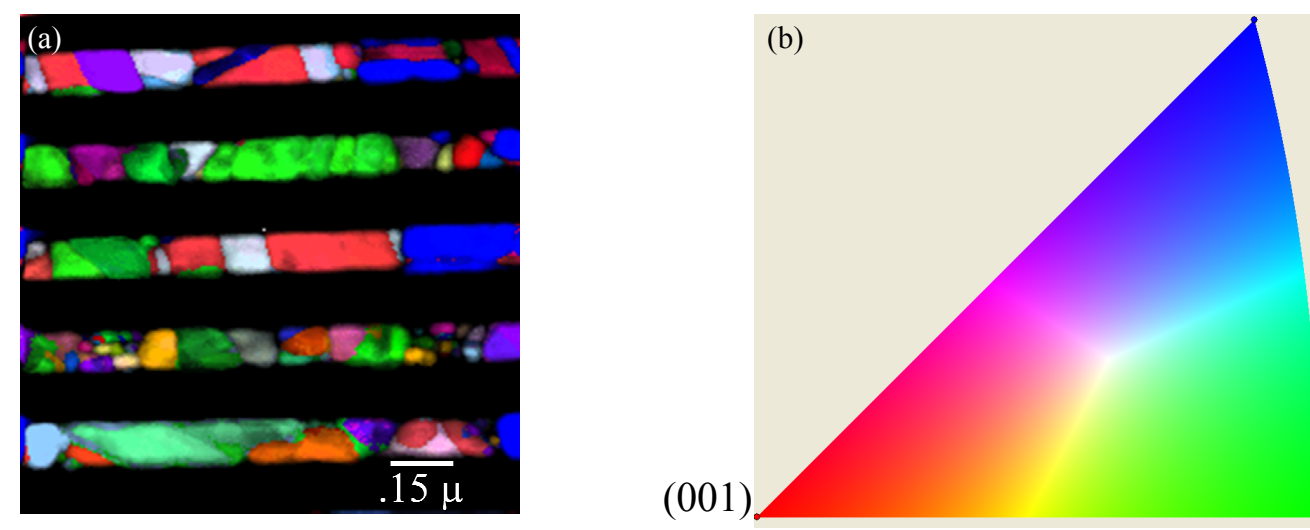

Figure 2: (a) Orientation map of CI grains obtained using D-STEM and ASTAR, (b) Color codes for orientations. 